\title{
Mean-Based Error Measures for Intermittent Demand Forecasting
}

\author{
S. D. Prestwich ${ }^{1}$, R. Rossi ${ }^{2}$, S. A. Tarim ${ }^{3}$, and B. Hnich ${ }^{4}$ \\ ${ }^{1}$ Department of Computer Science, University College Cork, Ireland \\ ${ }^{2}$ University of Edinburgh Business School, Edinburgh, UK \\ 32Department of Management, Hacettepe University, Ankara, Turkey \\ ${ }^{4}$ Computer Engineering Department, Izmir University of Economics, Turkey \\ s.prestwich@cs.ucc.ie, roberto.rossi@wur.nl, \\ armagan_tarim@hacettepe.edu.tr, brahim.hnich@ieu.edu.tr
}

\begin{abstract}
To compare different forecasting methods on demand series we require an error measure. Many error measures have been proposed, but when demand is intermittent some become inapplicable, some give counter-intuitive results, and there is no agreement on which is best. We argue that almost all known measures rank forecasters incorrectly on intermittent demand series. We propose several new error measures with wider applicability, and correct forecaster ranking on several intermittent demand patterns. We call these "mean-based" error measures because they evaluate forecasts against the (possibly time-dependent) mean of the underlying stochastic process instead of point demands.
\end{abstract}

\section{Introduction}

Inventory management is of great economic importance to industry, but forecasting demand for spare parts is difficult because it is intermittent: in many time periods the demand is zero. This type of demand occurs in several industries, for example in aerospace and military inventories from which spare parts such as wings or jet engines are infrequently required. Various methods have been proposed for forecasting, some simple and others statistically sophisticated, but relatively little work has been done on intermittent demand. Most work in this area is influenced by that of [5], who first separated the forecasting of demand size and inter-demand interval. A survey of forecasting methods for spare parts is given in 3 .

To choose a good forecasting method we can test the alternatives empirically on demand series to see which gives the smallest error. For this we require an error measure (or accuracy measure). Unfortunately, there is no general agreement on which of the many existing error measures is best. Forecasting methods have been extensively compared on real and simulated data in the well-known M-, M2- and M3-competitions [14[15]16] using several error measures. However, these forecasting competitions did not deal specifically with intermittent demands, so the experience gained from these competitions cannot be used as a guide. 
For intermittent demand series some error measures are inapplicable because division by zero leads to infinities, but there are still several possibilities. This is an important issue because if researchers are free to choose from a large set of measures then their results are likely to be incomparable. Moreover, there is a temptation to choose measures that give desired results [24], making experiments less objective. In 1992 the editor of the International Journal of Forecasting wrote that the choice of error measure is not a matter of personal preference and urged researchers to follow contemporary recommendations [24].

In this paper we examine the suitability of known error measures for intermittent demand, and propose new improved measures. Section 2 provides background, demonstrates anomalous behaviour in existing error measures, and proposes new measures. Section 3 evaluates the new measures on simulated data. Section 4 concludes the paper.

\section{New error measures}

In this section we provide some necessary background and describe our contribution. Section 2.1] describes the relevant forecasting methods, Section [2.2 surveys known error measures, Section 2.3 argues that these measures can rank forecasters incorrectly, and Section 2.4 proposes new measures.

\subsection{Forecasting methods}

First we describe the forecasting methods that will be used in the paper. Single exponential smoothing (SES) computes a smoothed series $\tilde{y}_{t}$ via the formula

$$
\tilde{y}_{t}=\alpha y_{t}+(1-\alpha) \tilde{y}_{t-1}
$$

where $\alpha \in(0,1)$ is a smoothing parameter. The smaller the value of $\alpha$ the less weight is attached to the most recent observations. An up-to-date survey of exponential smoothing algorithms is given in [8]. They perform remarkably well, often beating more complex approaches [7. However, SES is known to perform poorly on intermittent demand, at least under some error measures.

The standard method for handling intermittency is Croston's method [5] which applies SES to the non-zero demand sizes $y$ and inter-demand intervals $\tau$ independently, using smoothing factors $\alpha$ and $\beta$ respectively. Given smoothed demand $\tilde{y}_{t}$ and smoothed interval $\tilde{\tau}_{t}$ at time $t$, the forecast is $f_{t}=\tilde{y}_{t} / \tilde{\tau}_{t}$. Both $\tilde{y}_{t}$ and $\tilde{\tau}_{t}$, and hence $f_{t}$, are updated at each time $t$ for which $y_{t} \neq 0$. Alternative versions were proposed by 13/17/8 and we shall use the variant of Syntetos \& Boylan [18 which is known to have low bias and variance on stochastic demand.

We also mention the random walk method (RW), also known as the naive method: take the previous period's demand as a forecast. Though RW is a rather trivial forecaster, it is often used as a baseline for evaluating other methods.

Finally we mention the forecaster that always forecasts 0 , which following [20] we call ZF. It was proposed by Croston, mentioned by [25] and studied by 420 . 


\subsection{Existing error measures}

Next we survey error measures, largely based on 1011. No one error measure is generally accepted as useful on intermittent demand, and opinion is highly divided 24]. A common compromise is to use more than one measure as in the forecasting competitions, and [9] recommend using different measures for different types of demand.

Scale-dependent measures The most common are:

- Mean [Signed] Error (ME): mean $\left(e_{t}\right)$

- Mean Square Error (MSE): mean $\left(e_{t}^{2}\right)$

- Root Mean Square Error (RMSE): $\sqrt{\mathrm{MSE}}$

- Mean Absolute Error (MAE): mean $\left(\left|e_{t}\right|\right)$

- Median Absolute Error (MdAE): median $\left(\left|e_{t}\right|\right)$

where $e_{t}$ is the error $y_{t}-\hat{y}_{t}$. These are useful for comparing methods on one series, but not for comparing over several series. Doubt has been cast on the suitability of MAE for intermittent demand [26].

Percentage errors These are also popular:

- Mean Absolute Percentage Error (MAPE): mean $\left(\left|p_{t}\right|\right)$

- Median Absolute Percentage Error (MdAPE): median $\left(\left|p_{t}\right|\right)$

- Root Mean Square Percentage Error (RMSPE): $\sqrt{\operatorname{mean}\left(p_{t}^{2}\right)}$

- Root Median Square Percentage Error (RMdSPE): $\sqrt{\operatorname{median}\left(p_{t}^{2}\right)}$

- Symmetric Mean Absolute Percentage Error (sMAPE): mean $\left(200\left|e_{t}\right| /\left(y_{t}+\right.\right.$ $\left.\left.\hat{y}_{t}\right)\right)$

- Symmetric Median Absolute Percentage Error (sMdAPE): median(200| $e_{t} \mid /\left(y_{t}+\right.$ $\left.\left.\hat{y}_{t}\right)\right)$

where $p_{t}=100 e_{t} / y_{t}$. The last two measures are motivated by the fact that MAPE and MdAPE penalise positive errors more than negative ones.

However, percentage errors are undefined if any $y_{t}=0$ (and if $\hat{y}_{t}=0$ in the last two cases) and have very skewed distributions when $y_{t} \approx 0$. It is also pointed out in 11] that they assume a meaningful zero, which is not the case for some data such as temperatures. Despite these drawbacks MAPE is recommended by most textbooks and was the main error measure used in the M-competition, while MdAPE is recommended by [6], and sMAPE and sMdAPE were used in the M3-competition.

It is pointed out by [12] that many commercial software packages report a MAPE even when a series contains zeros, although the MAPE is technically undefined in this case. This is done by simply excluding periods with zero demands, which does not reflect the true errors of a forecast. We shall denote this MAPE variant by iMAPE. 
Relative error-based measures We may also scale by using errors from other measures:

- Mean Relative Absolute Error (MRAE): mean $\left(\left|r_{t}\right|\right)$

- Median Relative Absolute Error (MdRAE): median $\left(\left|r_{t}\right|\right)$

- Geometric Mean Relative Absolute Error (GMRAE): gmean $\left(\left|r_{t}\right|\right)$

where $r_{t}=e_{t} / e_{t}^{*}$ and $e_{t}^{*}$ is the error from a baseline method which is often RW.

GMRAE is also known as Relative Geometric Root Mean Square and has desirable statistical properties [6]. It is used by [18, and [1] recommends the use of relative error-based measures. It has been proposed for intermittent demand in particular [18. However, these measures have the drawback of infinite variance because $e_{t}^{*}$ can be arbitrarily small [4|11. In the particular case of intermittent demand with RW as baseline, $e_{t}^{*}$ is often zero so these measures are undefined. Extreme values can be trimmed [1] but this introduces some arbitrariness [1].

Relative measures Instead of computing an absolute quantity to measure the accuracy of a method, we may compare it with another method. This can be done for many types of error measure, for example

- Relative Mean Absolute Error (RelMAE): MAE/MAE ${ }_{b}$

- Relative Mean Squared Error (RelMSE): MSE/MSE

- Relative Root Mean Squared Error (RelRMSE): RMSE/RMSE

- etc

where $\mathrm{MAE}_{b}, \mathrm{MSE}_{b}$ and $\mathrm{RMSE}_{b}$ are the MAE, MSE and RMSE of a baseline measure. The most popular baseline is RW, in which case RelRMSE is Theil's U2 statistic 22 and log RelMSE is Thompson's LMR measure 23. RelMAE was recommended for intermittent demand by [18] and called CumMAE by [1]. It is unlikely for these measures to become infinite, because the denominator is only zero if the baseline forecaster gives perfect results.

Another relative measure is Percent Better (PB) where a method is compared to another, usually RW, by how often its absolute error is smaller. This is recommended by [12. A related measure is Percent Best (PBt) which compares several methods and computes the percentage of times each is most accurate. A drawback with PB and PBt is that they give no indication of the size of errors, so one large error is considered to be less serious than two tiny errors.

Scaled errors We mention two of these. Firstly, the MAD/Mean Ratio 12, also called the Weighted MAPE, which we shall abbreviate to MMR. MAD (Mean Absolute Deviation) is another name for MAE so MMR = MAE/ME. Secondly, the MASE [1] (Mean Absolute Scaled Error) defined by

$$
\operatorname{MASE}=\operatorname{mean}\left(\left|q_{t}\right|\right)
$$

where $q_{t}$ is a scaled error defined by

$$
q_{t}=\frac{e_{t}}{\frac{1}{n-1} \sum_{i=2}^{n}\left|y_{i}-y_{i-1}\right|}
$$


and $t=1 \ldots n$ is the set of sample periods used for forecasting. MASE effectively evaluates a forecasting method against RW. The only situation in which it is unusable is when all in-sample demands are identical. Other scaled error measures defined analogously to MASE include the Root Mean Squared Scaled Error (RMSSE) and the Median Absolute Scaled Error (MdASE). MASE has been argued to be superior to several other methods used in forecasting competitions. An advantage of MASE over MMR is that it is more reliable on demand with seasonality, trends or other forms of non-stationarity. However, [12 note that the MASE of two series with identical forecasts and identical demands during the forecast horizon will differ if the two series differed in their historical demands. This is counter-intuitive so MASE is not always easy to interpret.

\subsection{Ranking forecasters}

Which forecaster is best for intermittent demand? There is no universally-agreed ranking but $\mathrm{CR}$ is often applied in practice to intermittent demand [7], and versions of $\mathrm{CR}$ are used in leading statistical forecasting software packages such as SAP and Forecast Pro 21] so we might expect it to be ranked first. However, there is some debate on this issue.

4 investigate whether MAPE, MSE or U2 is the best error measure for intermittent demand, using more than one error measure. They find that ZF does surprisingly well, beating SES and CR on lumpy demand under a modified MAPE, but losing under MSE and U2. However, 20] note that ZF is of no practical use for inventory control. [18] found that SES beat CR on intermittent demand, using more than one error measure, though $\mathrm{CR}$ was better if issue point only were considered. According to [8] it is hard to conclude from the various studies that $\mathrm{CR}$ is best, because the results depend on the data and error measures used. 2] also note that there is no conclusive evidence pointing to a best method. But 20 conclude that the apparently poor performance of $\mathrm{CR}$ in some studies is caused by the use of inappropriate error measures, while 920127] found that CR beats SES on intermittent series.

Our position is that it is both reasonable and consistent with current wisdom to rank CR above SES, and SES above ZF, on intermittent demand series. CR has maintained its popularity over several decades, and if practitioners prefer a method based on experience, then any error measure that disagrees with this preference is of little use to them. We shall therefore take it as axiomatic that any error measure that fails this test, which we denote by $\mathrm{CR} \succ \mathrm{SES} \succ \mathrm{ZF}$, should not be applied to intermittent demand. Of course some researchers will disagree with this position, which is entirely reasonable, but we hope that our work will be of use to those who agree with our ranking axiom.

\subsection{Mean-based error measures}

In Section 3 we shall test known error measures against this axiom. First we propose several new measures: in fact one for each existing measure, obtained by evaluating forecasts against the mean of the underlying stochastic process of 
the demand, which we denote $y_{t}^{m}$, instead of the point demand $y_{t}$. So instead of the usual error $e_{t}=y_{t}-\hat{y}_{t}$ we use $e_{t}^{m}=y_{t}^{m}-\hat{y}_{t}$, instead of $p_{t}=100 e_{t} / y_{t}$ we use $p_{t}^{m}=100 e_{t}^{m} / y_{t}^{m}$, and similarly for baseline measures. The error $e_{t}^{m}$ measures how well a forecaster ignores noise and estimates the underlying demand rate. We shall call these mean-based measures, and denote them by adding the prefix " $\mathrm{m}$ " to the measure they are based on (mMAE, mMSE, etc).

For artificial data it is easy to find $y_{t}^{m}$. For stationary demand we can use $y_{t}^{m}=$ mean $\left(y_{t}\right)$ where the mean is either derived analytically or simply computed over the entire series. For non-stationary demand the mean of the stochastic process is a function of time, but we can still use our knowledge of the data to obtain the dynamic underlying demand rate. For example the obsolescence experiments of 21] use artificial data whose non-zero demand probability drops either linearly or abruptly to 0 , whereas demand sizes follow a fixed distribution: in either case we can multiply the current probability by the fixed distribution mean to obtain $y_{t}^{m}$.

On real-world series the stochastic process is of course unknown, though one can make assume a particular form (for example a Poisson process) then estimate its parameters. A simple approach is to estimate the current mean demand via standard techniques used to estimate seasonal components. It is common to estimate a seasonal component by taking a moving average over a window, stretching forward and backward in time. We can use the same moving window technique to obtain a smoothed version of the demand series, and use this as $y_{t}^{m}$. If the demand series is too short to use a moving window, we can estimate the changing mean by regression. Or if we assume demand to be stationary, we can take the series mean as $y_{t}^{m}$. As with seasonal component estimation, there are several reasonable approaches.

Mean-based error measures have wider applicability than their original counterparts. Percentage errors such as MAPE are undefined whenever $y_{t}=0$, whereas mMAPE is only undefined when $y_{t}^{m}=0$ : for stationary demand $y_{t}^{m}$ is the series mean, which is only zero when $y_{t}=0$ for all $t$. Relative error-based measures such as GMRAE with RW as baseline are undefined on intermittent demand, because both the demand and RW's forecast are often zero so the RW error $e_{t}^{*}$ (which is the denominator) is also zero. However, a measure such as mGMRAE is only undefined when the denominator $e_{t}^{* m}=y_{t}^{* m}-\hat{y}_{t}^{*}$ is zero: the RW forecast $\hat{y}_{t}^{*}$ will often be zero, but again $y_{t}^{* m}$ is only zero when $y_{t}=0$ for all $t$.

We now have a large number of new error measures, none of which is likely to give infinite answers on intermittent demand. This allows us to measure forecasting deviations using absolute or squared values, in scaled or unscaled ways, and on one or multiple demand series. In the next section we shall evaluate them with respect to our forecaster ranking axiom. 


\section{Experiments}

The error measures we compare are selected from the various classes described in Section 2.2. To represent the scale-dependent measures we use MAE, MdAE and MSE and their mean-based equivalents mMAE, mMdAE and mMSE; from the percentage errors we use iMAPE and mMAPE. Note that the mMAPE of $\mathrm{ZF}$ is always $100 e_{t}^{m} / y_{t}^{m}=100\left(y_{t}^{m}-0\right) / y_{t}^{m}=100$ and the iMAPE of ZF is always $100 e_{t} / y_{t}=100\left(y_{t}-0\right) / y_{t}=100(\mathrm{MAPE}$ is undefined on intermittent demand). To represent the relative error-based measures we use only mGMRAE with RW as baseline (GMRAE with RW as baseline is undefined for intermittent demand). To represent the relative measures we use $\mathrm{PB}$ and $\mathrm{mPB}$ with $\mathrm{RW}$ as baseline.

We start with data based on that used in the experiments of Teunter et al. 21. Demands occur with some probability in each period, hence inter-demand intervals are distributed geometrically, and we use a logarithmic distribution for demand sizes. Geometrically distributed intervals are a discrete version of Poisson intervals, and the combination of Poisson intervals and logarithmic demand sizes yields a negative binomial distribution, for which there is theoretical and empirical evidence: see for example the recent discussion in [19. Teunter et al. generate demand data that is nonzero with probability $p_{0}$ where $p_{0}$ is either 0.2 or 0.5 , and whose size is logarithmically distributed. The logarithmic distribution is characterised by a parameter $\ell \in(0,1)$ and is discrete with $\operatorname{Pr}[X=k]=-\ell^{k} / k \log (1-\ell)$ for $k \geq 1$. They use two values: $\ell=0.001$ to simulate low demand and $\ell=0.9$ to simulate lumpy demand.

Tables 14 show best results for SES, CR and ZF using $\alpha$ and $\beta$ values chosen from $\{0.1,0.2,0.3\}$. We initialise the forecasters by choosing arbitrary initial values $\hat{y}_{0}=\hat{\tau}_{0}=1$ then running them for $10^{4}$ periods using demand probability $p_{0}$. Results are then computed over $10^{5}$ time periods. To estimate the stochastic process mean we simply compute the mean of all $10^{5}$ demands (including zeros). The results show that MAE, MdAE and iMAPE are unreliable error measures for some types of intermittent demand because they incorrectly rank the three forecasters. PB is more reliable but the differences are sometimes very small, and in one case PB ranked CR and SES equally. Among existing measures only MSE behaves correctly. However, all mean-based measures behave correctly, though $\mathrm{mPB}$ still scores CR and SES quite similarly. We also tried geometrically distributed demand sizes as in 21], and regular intermittent demand as in [5], with similar results.

However, in further experiments MSE was also unreliable. Willemain et al. [27] point out that demand in industrial data is often autocorrelated: demand may occur in streaks, with longer sequences of zero or nonzero values than one would expect. This is a positive autocorrelation on demand intervals, but they also observed negative autocorrelation: frequent alternation between zero and nonzero demand. Following Willemain et al. we model autocorrelation by a firstorder 2-state Markov process. Let all demands be 0 or 1 , and denote the transition probability from 0 to 1 by $p_{01}$, and from 1 to 0 by $p_{10}$. On negatively autocorrelated demand MSE ranks correctly, but results for positively autocor- 


\begin{tabular}{|c|c|c|c|c|c|c|}
\hline error & & SES & & $\mathrm{CR}$ & $\mathrm{ZF}$ & forecaster \\
\hline mea & $\alpha$ & error & $\alpha$ & error & error & \\
\hline MAE & 0.3 & 0.32134 & $\begin{array}{lll}0.1 & 0.3\end{array}$ & 0.31846 & 0.20141 & $\mathrm{ZF} \succ \mathrm{CR} \succ \mathrm{SES}$ \\
\hline MdAE & 0.3 & 0.23740 & $\begin{array}{lll}0.3 & 0.3\end{array}$ & 0.20867 & 0.00000 & $\mathrm{ZF} \succ \mathrm{CR} \succ \mathrm{SES}$ \\
\hline $\mathrm{SE}$ & 0.1 & 0.1 & & 0.1 & 0.20151 & $\mathrm{CR} \succ \mathrm{SES} \succ \mathrm{ZF}$ \\
\hline iMAPE & 0.3 & 79.77339 & 0.10 .1 & 80.07155 & 100.00000 & $\mathrm{SES} \succ \mathrm{CR} \succ \mathrm{ZF}$ \\
\hline $\mathrm{PB}$ & 0. & 02.02000 & 0.10 .1 & 32.52000 & 16.26000 & $\mathrm{CR}=\mathrm{SES} \succ \mathrm{ZF}$ \\
\hline mMAE & 0.1 & 0.07434 & $\begin{array}{ll}0.1 & 0.1\end{array}$ & 0.03225 & 0.20122 & $\mathrm{CR} \succ \mathrm{SES} \succ \mathrm{ZF}$ \\
\hline $\mathrm{mMdAE}$ & 0.3 & 0.13379 & 0.30 .3 & 0.04651 & 0.20160 & $\mathrm{CR} \succ \mathrm{SES} \succ \mathrm{ZF}$ \\
\hline mMSE & 0.1 & 0.00856 & 0.10 .1 & 0.00167 & 0.04054 & $\mathrm{CR} \succ \mathrm{SES} \succ \mathrm{ZF}$ \\
\hline mMAPE & 0.1 & 36.91216 & 0.10 .1 & 16.01403 & 100.00000 & $\mathrm{CR} \succ \mathrm{SES} \succ \mathrm{ZF}$ \\
\hline $\mathrm{PB}$ & 0. & 97.83 & 0.10 .3 & 98.7 & 20.15000 & $\succ \mathrm{SES} \succ \mathrm{ZF}$ \\
\hline mGMRAE & 0.1 & 0.30005 & 0.10 .1 & 0.13512 & 0.84936 & $\succ \mathrm{SES} \succ \mathrm{ZF}$ \\
\hline
\end{tabular}

Table 1. Results for artificial demand with $p_{0}=0.2$ and $\ell=0.001$

\begin{tabular}{|c|c|c|c|c|c|c|}
\hline error & & SES & & CR & $\mathrm{ZF}$ & \\
\hline ure & $\alpha$ & error & & error & error & \\
\hline$\overline{\mathrm{MAE}}$ & 0.3 & 0.49945 & $\begin{array}{lll}0.1 & 0.3\end{array}$ & 0.49962 & 0.49963 & $\mathrm{SES} \succ \mathrm{CR} \succ \mathrm{ZF}$ \\
\hline & & 0.50 & 0.3 & 44 & 0000 & $\mathrm{ZF} \succ \mathrm{CR} \succ \mathrm{SES}$ \\
\hline & & 0.26335 & 10.1 & 0.25643 & 0.50003 & $\succ \mathrm{SES} \succ \mathrm{ZF}$ \\
\hline & & 49 & & 3300 & 100. & $\mathrm{CR} \succ \mathrm{SE}$ \\
\hline $\mathrm{PB}$ & 0.3 & 50.63000 & 0.10 .1 & 50.65000 & 25.31000 & $\mathrm{CR} \succ \mathrm{SES} \succ \mathrm{ZF}$ \\
\hline mMAE & 0.1 & 0.09310 & 0.10 .1 & 0.06324 & 0.49993 & $\succ \mathrm{SES} \succ \mathrm{ZF}$ \\
\hline & 0. & 0.1511 & 0.30 .3 & 0.096 & 0.49870 & $\succ \mathrm{SES} \succ \mathrm{ZF}$ \\
\hline & 0.1 & 0.013 & 0.10 .1 & 0.00614 & 0.24985 & $\succ \mathrm{SES} \succ \mathrm{ZF}$ \\
\hline APE & 0. & & 0.10 .1 & 12.65683 & 100.00000 & $\mathrm{CR} \succ \mathrm{SES} \succ \mathrm{ZF}$ \\
\hline & & 99.950 & 0.10 .1 & 100.00000 & 49.84000 & $\mathrm{CR} \succ \mathrm{SES} \succ \mathrm{ZF}$ \\
\hline mGMRAE & 0.1 & 0.17928 & 0.10 .1 & 0.12201 & 0.99721 & $\succ \mathrm{SES} \succ \mathrm{ZF}$ \\
\hline
\end{tabular}

Table 2. Results for artificial demand with $p_{0}=0.5$ and $\ell=0.001$

\begin{tabular}{|c|c|c|c|c|c|}
\hline \multirow{2}{*}{$\begin{array}{l}\text { error } \\
\text { measure }\end{array}$} & \multicolumn{2}{|r|}{ SES } & $\mathrm{CR}$ & \multirow{2}{*}{\begin{tabular}{r|}
$\mathrm{ZF}$ \\
error
\end{tabular}} & \multirow{2}{*}{$\begin{array}{c}\text { forecaster } \\
\text { ranking }\end{array}$} \\
\hline & $\alpha$ & error & error & & \\
\hline MAE & 0.1 & 1.25741 & 1.23205 & 0.77191 & $\mathrm{ZF} \succ \mathrm{CR} \succ \mathrm{SES}$ \\
\hline MdAE & 0.3 & 0.53944 & 0.67425 & 0.00000 & $\mathrm{ZF} \succ \mathrm{SES} \succ \mathrm{CR}$ \\
\hline MSE & 0.1 & 7.10279 & $\begin{array}{lll}0.1 & 0.1 & 6.83258\end{array}$ & 7.35755 & $\mathrm{CR} \succ \mathrm{SES} \succ \mathrm{ZF}$ \\
\hline iMAPE & 0.1 & 68.79125 & 0.10 .159 .83256 & 100.00000 & $\mathrm{CR} \succ \mathrm{SES} \succ \mathrm{ZF}$ \\
\hline $\mathrm{PB}$ & 0.3 & 31.80000 & 0.10 .333 .09000 & 17.31000 & $\mathrm{CR} \succ \mathrm{SES} \succ \mathrm{ZF}$ \\
\hline mMAE & 0.1 & 0.44085 & $\begin{array}{|lll|}0.1 & 0.1 & 0.21087\end{array}$ & 0.77266 & $\mathrm{CR} \succ \mathrm{SES} \succ \mathrm{ZF}$ \\
\hline $\mathrm{mMdAE}$ & 0.3 & 0.56279 & 0.28997 & 0.76560 & $\mathrm{CR} \succ \mathrm{SES} \succ \mathrm{ZF}$ \\
\hline mMSE & 0.1 & 0.36904 & $\begin{array}{lll}0.1 & 0.1 & 0.07450\end{array}$ & 0.59660 & $\mathrm{CR} \succ \mathrm{SES} \succ \mathrm{ZF}$ \\
\hline mMAPE & 0.1 & 57.11138 & 0.10 .127 .31830 & 100.00000 & $\mathrm{CR} \succ \mathrm{SES} \succ \mathrm{ZF}$ \\
\hline $\mathrm{mPB}$ & 0.2 & 87.12000 & 0.30 .389 .02000 & 12.42000 & $\mathrm{CR} \succ \mathrm{SES} \succ \mathrm{ZF}$ \\
\hline mGMRAE & 0.1 & 0.58574 & $\begin{array}{lll}0.1 & 0.1 & 0.29300\end{array}$ & 1.09061 & $\mathrm{CR} \succ \mathrm{SES} \succ \mathrm{ZF}$ \\
\hline
\end{tabular}

Table 3. Results for artificial demand with $p_{0}=0.2$ and $\ell=0.9$ 


\begin{tabular}{|c|c|c|c|c|c|c|}
\hline \multirow{2}{*}{$\begin{array}{l}\text { error } \\
\text { measure }\end{array}$} & \multicolumn{2}{|r|}{ SES } & \multicolumn{2}{|c|}{$\mathrm{CR}$} & \multirow{2}{*}{$\begin{array}{r}\mathrm{ZF} \\
\text { error }\end{array}$} & \multirow{2}{*}{$\begin{array}{c}\text { forecaster } \\
\text { ranking }\end{array}$} \\
\hline & $\alpha$ & error & $\alpha$ & error & & \\
\hline MAE & 0.1 & 2.38007 & $\begin{array}{lll}0.1 & 0.3 \\
\end{array}$ & 2.28662 & 1.93788 & $\overline{\mathrm{ZF}} \succ \mathrm{CR} \succ \mathrm{SES}$ \\
\hline MdAE & 0.3 & 1.45094 & 0.30 .3 & 1.38990 & 0.00000 & $\mathrm{ZF} \succ \mathrm{CR} \succ \mathrm{SES}$ \\
\hline MSE & 0.1 & 16.01983 & 0.10 .1 & 15.59856 & 18.97148 & $\mathrm{CR} \succ \mathrm{SES} \succ \mathrm{ZF}$ \\
\hline iMAPE & 0.1 & 72.02 & 0.10 .3 & 65.44701 & 100.00000 & $\mathrm{CR} \succ \mathrm{SES} \succ \mathrm{ZF}$ \\
\hline $\mathrm{PB}$ & 0.1 & 50.78000 & 0.10 .1 & 50.89000 & 31.69000 & $\mathrm{CR} \succ \mathrm{SES} \succ \mathrm{ZF}$ \\
\hline mMAE & 0.1 & 0.68463 & 0.10 .1 & 0.48617 & 1.93752 & $\mathrm{CR} \succ \mathrm{SES} \succ \mathrm{ZF}$ \\
\hline $\mathrm{mMdAE}$ & 0.3 & 0.95963 & 0.30 .3 & 0.72759 & 1.90290 & $\mathrm{CR} \succ \mathrm{SES} \succ \mathrm{ZF}$ \\
\hline mMSE & 0.1 & 0.79809 & 0.10 .1 & 0.38147 & 3.75220 & $\mathrm{CR} \succ \mathrm{SES} \succ \mathrm{ZF}$ \\
\hline mMAPE & 0.1 & 35.32882 & 0.10 .1 & 25.08728 & 100.00000 & $\mathrm{CR} \succ \mathrm{SES} \succ \mathrm{ZF}$ \\
\hline $\mathrm{mPB}$ & 0.3 & 78.51000 & 0.30 .3 & 79.11000 & 15.88000 & $\mathrm{CR} \succ \mathrm{SES} \succ \mathrm{ZF}$ \\
\hline mGMRAE & 0.1 & 0.94599 & 0.10 .1 & 0.72287 & 2.83995 & $\mathrm{CR} \succ \mathrm{SES} \succ \mathrm{ZF}$ \\
\hline
\end{tabular}

Table 4. Results for artificial demand with $p_{0}=0.5$ and $\ell=0.9$

related demand with $p_{01}=p_{10}=0.3$ are shown in Table 5. Here MAE, MdAE, MSE and iMAPE are all unreliable while mMAE, mMdAE, mMSE, mMAPE, mGMRAE and $\mathrm{mPB}$ give correct rankings, as does $\mathrm{PB}$. We found similar results for other values of $p_{01}$ and $p_{10}$.

\begin{tabular}{|c|c|c|c|c|c|c|}
\hline & & SES & & $\mathrm{R}$ & $\mathrm{ZF}$ & \\
\hline & $\alpha$ & error & $\alpha \beta$ & error & error & \\
\hline$\overline{\mathrm{MAE}}$ & 0.3 & 0.41 & $\begin{array}{lll}0.1 & 0.3\end{array}$ & & 9880 & $\mathrm{SES} \succ \mathrm{ZF}$ \\
\hline & & 0.377 & 30.3 & 0.49221 & .00000 & $\succ \mathrm{SES} \succ c$ \\
\hline & & & & & & $\mathrm{SES} \succ \mathrm{CR} \succ \mathrm{ZH}$ \\
\hline E & 0.3 & 41.77340 & 0.10 .1 & 49.86910 & 100.000 & $\mathrm{SES} \succ \mathrm{CR} \succ \mathrm{ZF}$ \\
\hline B & 0.3 & $t$ & 0.1 & 44.29000 & 21.82000 & $\mathrm{CR} \succ \mathrm{SES} \succ \mathrm{ZF}$ \\
\hline $\mathrm{mM}$ & & 0.13732 & 0.10 .1 & 0.09385 & 0.49902 & $\succ \mathrm{SES} \succ \mathrm{ZF}$ \\
\hline & & 0 & 0.0 & & & $\succ \mathrm{SES} \succ \mathrm{ZF}$ \\
\hline & & 0.028 & & & 4865 & $\mathrm{CR} \succ \mathrm{SES} \succ \mathrm{ZF}$ \\
\hline & & 27.5 & 0.1 & 18.8 & 0.000 & $\mathrm{CR} \succ \mathrm{SES} \succ \mathrm{ZF}$ \\
\hline & & & & & 30.0 & $\mathrm{CR} \succ \mathrm{SES} \succ \mathrm{ZF}$ \\
\hline aGMRA & 0.1 & 0.28674 & 0.10 .1 & 0.20195 & 0.99883 & $\mathrm{CR} \succ \mathrm{SES} \succ \mathrm{ZF}$ \\
\hline
\end{tabular}

Table 5. Results for autocorrelated demand with $p_{01}=p_{10}=0.3$

Collectively these results imply that almost all tested error measures are unreliable. They also imply that other untested error measures are unreliable, because they are monotonic functions of MAE or MSE and hence rank forecasters in the same way. These include RMSE, relative measures such as RelMAE and RelMSE and as their special cases U2 and LMR, and scaled errors such as MMR and MASE. Hence most existing error measures are inapplicable to intermittent 
demand, if our ranking axiom is reasonable. PB was almost correct, but in one case it was unable to distinguish between CR and SES (see Table 1).

We conclude that all known error measures (except ME which measures bias, not deviation) are unreliable on some types of intermittent demand, even when they do not incur infinities, so there is currently no reliable way of measuring deviation. These results reinforce and complement those of [20, who show that MAE and RMSE rank ZF above SES (and above a moving average), and SES above CR, on a large data set of intermittent demand from an air force. Our results apply to more error measures and are more easily reproducible, being based on simple artificial data. But their result shows that the inappropriateness of at least some current error measures extends to real data. In contrast, our new measures gave correct results in all cases.

Based on our experiments, and on inapplicabilities pointed out by other researchers, we make two proposals regarding measures of deviation for intermittent demand. Firstly, we do not recommend any existing error measures. Secondly, we recommend several new error measures: mMSE, mRMSE, mMAE, mMdAE, mMAPE, mRelMSE (including special cases mU2 and mLMR plus other relative measures such as mRelMAE), mRelRMSE, mMMR, mMASE and mGMRAE. Which of these is best depends on user preference, and considerations such as whether errors are to be compared on one or across several series.

In our experiments we did not evaluate several other possible new measures: mMdAPE, mRMSPE, mRMdSPE, msMAPE, msMdAPE, mMRAE, mMdRAE, mRMSSE and mMdASE, all defined in the obvious way. We leave their evaluation for future work.

\section{Conclusion}

We have shown that almost all known error measures rank forecasting methods incorrectly on some intermittent demand series. Given this result, and the wellknown fact that several error measures are inapplicable to intermittent demand because of infinities, there is currently no reliable way of measuring forecast deviation errors on such demands.

To alleviate this problem, we described a simple way of modifying all known error measures so that they are more widely applicable and behave more correctly. This yields many new mean-based error measures that can be used to compare forecasters on intermittent demand. They are unlikely to be plagued by infinities, and in tests they consistently ranked forecasters correctly.

We have defined a large number of new error measures, and it might be argued that this only adds to the confusion. But we believe that the improved behaviour and wider applicability of our measures make them worth considering when faced with intermittent demand. To simplify matters we should perhaps recommend a small number of new measures. Based on popularity and the recommendations of experts, we choose the mean-based analogues of MAPE, GMRAE and U2.

In future work we shall evaluate other error measures that can be modified by our technique. We shall experiment with artificial non-stationary data and 
real-world series. The statistical properties of the new error measures should be investigated. Finally, the new error measures can also be applied to nonintermittent demand, and we shall evaluate their usefulness using series from the forecasting competitions.

Acknowledgment This work was partially funded by Enterprise Ireland Innovation Voucher IV-2009-2092.

\section{References}

1. J. S. Armstrong, F. Collopy. Error Measures for Generalizing About Forecasting Methods: Empirical Comparisons. International Journal of Forecasting 8:69-80, 1992.

2. A. Bacchetti, N. Saccani. Spare Parts Classification and Demand Forecasting for Stock Control: Investigating the Gap Between Research and Practice. Omega 40(6):722-737, 2012.

3. J. E. Boylan, A. A. Syntetos. Spare Parts Management: a Review of Forecasting Research and Extensions. IMA Journal of Management Mathematics 21(3):227237, 2010.

4. D. C. Chatfield, J. C. Hayyab. All-Zero Forecasts for Lumpy Demand: a Factorial Study. International Journal of Production Research 45(4):935-950, 2007.

5. J. D. Croston. Forecasting and Stock Control for Intermittent Demands. Operational Research Quarterly 23:289-304, 1972.

6. R. Fildes. The Evaluation of Extrapolative Forecasting Methods. International Journal of Forecasting 8(1):81-98, 1992.

7. R. Fildes, K. Nikolopoulos, S. F. Crone, A. A. Syntetos. Forecasting and Operational Research: a Review. Journal of the Operational Research Society 59:1150$1172,2008$.

8. E. S. Gardner Jr. Exponential Smoothing: the State of the Art - Part II. International Journal of Forecasting 22(4):637-666, 2006.

9. A. A. Ghobbar, C. H. Friend. Evaluation of Forecasting Methods for Intermittent Parts Demand in the Field of Aviation: a Predictive Model. Computers \& Operations Research 30:2097-2114, 2003.

10. J. D. de Gooijer, R. J. Hyndman. 25 Years of IIF Time Series Forecasting: a Selective Review, Tinbergen Institute Discussion Paper No 05-068/4, Tinbergen Institute, 2005.

11. R. J. Hyndman, A. B. Koehler. Another Look at Measures of Forecast Accuracy. International Journal of Forecasting 22(4):679-688, 2006.

12. S. Kolassa, W. Schütz. Advantages of the MAD/Mean Ratio Over the MAPE. Foresight: the International Journal of Applied Forecasting 6:40-43, 2007.

13. E. Levén, A. Segerstedt. Inventory Control With a Modified Croston Procedure and Erlang Distribution. International Journal of Production Economics 90(3):361367, 2004.

14. S. Makridakis, A. Andersen, R. Carbone, R. Fildes, M. Hibon, R. Lewandowski, J. Newton, E. Parzen R. Winkler. The Accuracy of Extrapolation (Time Series) Methods: Results of a Forecasting Competition. Journal of Forecasting 1:111-153, 1982. 
15. S. Makridakis, C. Chatfield, M. Hibon, M. Lawrence, T. Mills, K. Ord, L. F. Simmons. The M-2 Competition: a Real-Time Judgmentally Based Forecasting Study. International Journal of Forecasting 9:5-23, 1993.

16. S. Makridakis, M. Hibon. The M3-Competition: Results, Conclusions and Implications. International Journal of Forecasting 16(4):451-476, 2000.

17. A. A. Syntetos. Forecasting for Intermittent Demand. Unpublished PhD thesis, Buckinghamshire Chilterns University College, Brunel University, 2001.

18. A. A. Syntetos, J. E. Boylan. The Accuracy of Intermittent Demand Estimates. International Journal of Forecasting 21:303-314, 2005.

19. A. Syntetos, Z. Babai, D. Lengu, N. Altay. Distributional Assumptions for Parametric Forecasting of Intermittent Demand. In: N. Altay \& A. Litteral (eds.), Service Parts Management: Demand Forecasting and Inventory Control, Springer Verlag, NY, USA, 2011, pp.31-52.

20. R. H. Teunter, L. Duncan. Forecasting Intermittent Demand: a Comparative Study. Journal of the Operational Research Society 60:321-329, 2009.

21. R. Teunter, A. A. Syntetos, M. Z. Babai. Intermittent Demand: Linking Forecasting to Inventory Obsolescence. European Journal of Operations Research 214:606-615, 2011.

22. H. Thiel. Applied Economic Forecasting. Rand McNally, 1966.

23. P. A. Thompson. An MSE Statistic for Comparing Forecast Accuracy Across Series. International Journal of Forecasting 6:219-227, 1990.

24. Various authors. A Commentary on Error Measures. International Journal of Forecasting 8:99-111, 1992.

25. G. H. K. Venkitachalam, D. B. Pratt, C. F. DeYoung, S. A. Morris, M. L. Goldstein. Forecasting and Inventory Planning for Parts With Intermittent Demand - a Case Study. Presented at the Industrial Engineering Research Conference, Portland, OR USA, 2003.

26. P. Wallström, A. Segerstedt. Evaluation of Forecasting Error Measurements and Techniques for Intermittent Demand. International Journal of Production Economics 128(2):625-636, 2010.

27. T. R. Willemain, C. N. Smart, J. H. Shockor, P. A. DeSautels. Forecasting Intermittent Demand in Manufacturing: a Comparative Evaluation of Croston's Method. International Journal of Forecasting 10(4):529-538, 1994. 